\title{
Mitochondrial Dynamics in Tachycardiomyopathy
}

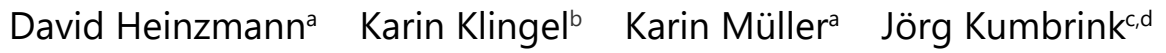 \\ Thomas Kirchner,d Jürgen Schreieck ${ }^{\mathrm{a}} \quad$ Meinrad Gawaz ${ }^{\mathrm{a}} \quad$ Peter Seizer $^{\mathrm{a}}$
}

aDepartment of Cardiology and Cardiovascular Diseases, University of Tübingen, Tübingen, Germany, ${ }^{b}$ Cardiopathology, Institute of Pathology and Neuropathology, University of Tübingen, Tübingen, Germany, Institute of Pathology, Faculty of Medicine, LMU Munich, Munich, Germany, ' ${ }^{\mathrm{d}}$ German Cancer Consortium, German Cancer Research Center, Heidelberg, Germany

\section{Key Words}

Tachycardiomyopathy $\bullet \mathrm{TCM} \cdot$ Heart failure $\bullet$ Mitochondria $\bullet$ Mfn1 $\bullet$ Mfn2 $・$ Fusion

\begin{abstract}
:
Background/Aims: Tachycardiomyopathy (TCM) is a largely reversible form of non-ischemic heart failure. The underlying mechanism are, however, still today poorly understood. Recent data indicate distinct changes in mitochondrial distribution in these patients, compared to other non-ischemic cardiomyopathies.This study investigated underlying mechanisms in mitochondrial dynamics in endomyocardial biopsy samples (EMB) from patients with TCM and compared them to patients with dilated cardiomyopathy (DCM), which show similar clinical features. Methods: Focused mRNA analyses were performed on routinely obtained paraffinfixed EMB specimen from patients fulfilling TCM diagnosis criteria, as well as patients with DCM to elucidate regulatory changes in mitochondrial fusion, fission and mitophagy. Results: In patients with TCM we were able to identify mRNA of Mitofusin 1 and 2, two effector proteins regulating mitochondrial fusion, to be strongly upregulated compared to patients with DCM. Conclusively, we did not find differences in the mRNA expression of mitochondrial fission regulators including DRP1, Fis1, MFF, MiD49, and MiD51. Furthermore, we did not find significant changes in PINK1 expression, an important mediator for mitochondrial autophagy. Conclusion: The mRNA upregulation of Mitofusin 1 and 2 provides first insight into the complex changes of mitochondrial dynamics in cardiomyocytes of patients with reversible heart failure due to TCM.

\section{Introduction}

Tachycardic rhythm disturbances can lead to a reversible impairment of left ventricular ejection fraction (LVEF). While the diagnosis of tachycardiomyopathy (TCM) has, until recently, been a retrospective diagnosis, new evidence from human endomyocardial biopsies (EMB) gives us unique insight into the underlying pathophysiological changes and puts TCM 
into context of non-ischemic cardiomyopathies [1]. From a clinical perspective, patients with TCM often display properties of a dilated cardiomyopathy (DCM), yet have a fundamentally better prognosis with a significant improvement of LVEF after rhythm or rate control.

Our group has previously described a macrophage-dominated myocardial inflammation with a low, yet noticeable extend of fibrosis in these patients. Furthermore, we have noticed a disturbed mitochondrial distribution pattern within the cardiomyocytes of patients with TCM resulting in an enrichment of mitochondria at intercalated discs (EMID-sign) [1].

With an enhanced energy demand, preservation of mitochondrial homeostasis through mitochondrial fusion and fission is of fundamental importance for the regeneration of ATP. In the myocardium, mitochondria therefore undergo frequent fusion and fission, ensuring adaptation to increased energy demand, as well as regulation of mitophagy and induction of apoptosis. Hence, fusion and fission are tightly regulated, ensuring optimal energy distribution within cardiomyocytes. Dysregulation of mitochondrial dynamics results in cardiomyocyte dysfunction and death, resulting in heart failure [2,3]. Underlying mechanisms of the altered distribution of mitochondria in TCM have not been elucidated, yet. In this study, we describe specific changes in mRNA expression of pivotal regulators of mitochondrial dynamics that distinguish patients with TCM from patients diagnosed with DCM.

\section{Materials and Methods}

mRNA was extracted from paraffin-embedded EMB specimen form patients with TCM (fulfilling published clinical diagnostic criteria, which included heart rate at admission $>100$ beats/min, heart rhythm other than sinus rhythm, a recovery of LVEF after rhythm or rate control, and exclusion of other causes of heart failure [1], n=13) and DCM (fulfilling classification criteria from the American Heart Association and the European Society of Cardiology, $\mathrm{n}=10$ ) using an RNeasy FFPE Kit (Qiagen, Hilden, Germany) according to the manufacturer's instructions. The samples were analyzed using a nCounter assay (NanoString Technologies, Seattle, WA, USA) analyses and quality controls were performed according to the recommendations of the manufacturer. The study protocol has been approved by the committee on human research of the EberhardKarls University of Tübingen.

Variables are expressed as mean \pm SEM. Statistical analyses were done using Student $\mathrm{t}$ test (Prism 7, GraphPad Software, La Jolla, CA, USA). Differences were considered statistically significant if $\mathrm{p}<0.05$.

\section{Results}

Two key players have been identified as fundamental effector proteins enabling mitochondrial fusion. Mitofusin 1 and 2 (Mfn1 and 2, respectively) form homo-, as well as heterodimers, achieving fusion of outer mitochondrial membranes (OMM).

We found the expression of Mfn1 to be increased in patients with TCM compared to patients with DCM ( $<<0.01$, Fig. 1A). Similarly, Mfn2 expression was enhanced in patients with TCM as well, compared to DCM ( $\mathrm{p}<0.05$, Fig. 1B), suggesting a higher mitochondrial fusion rate.

Notably, PGC1 $\alpha$ as a known upstream regulator of Mfn1 and 2 did not show a difference in expression between the two groups ( $p>0.05$, Fig. 1C).

Dynamin-related protein1 (Drp1), as the main fission factor, is anchored via Fis1, MFF, MiD49, and MiD51 to the OMM, forming a multimeric structure to achieve membrane constriction and scission, resulting in fission of the mitochondrion. Drp1 expression was similar in patients with TCM and DCM, indicating similar fission rates (Fig. 1E). In line with this finding, the aforementioned immediate interaction partners showed no significant differences between the groups (Fig. 1F-H).

Mitophagy is a tightly regulated process eliminating dysfunctional mitochondria. In stress-induced mitochondrial depolarization, the reduced degradation of the mitochondrial kinase PINK1 leads to the increased attraction of autophagosomes resulting in mitophagy 
of damaged mitochondria. We found that the expression of PINK1 did not differ in patients with TCM compared to DCM (Fig. 1D).

\section{Discussion}

The described data above provides insight into differences in gene expression of pivotal regulation and effector proteins for mitochondrial fusion, fission, and mitophagy in patients with TCM.

We recently defined the EMID-sign as a distinct distribution pattern of mitochondria, which is found in patients with TCM [1]. Mitochondria, as the main ATP regeneration source in cardiomyocytes, are in constant interaction with each other. A careful balance of fusion and fission allows for adaptation to various stimuli, exchange of proteins and mtDNA, mitophagy, and apoptosis.

We provide evidence that enhanced mitochondrial fusion through higher abundance of Mfn1 and Mfn2 could be a pathophysiologically important mechanism of abnormal mitochondrial distribution in TCM. While the correlation of mRNA expression with the subsequent change in protein abundance is influenced by post-transcriptional and posttranslational modifications, Mfn
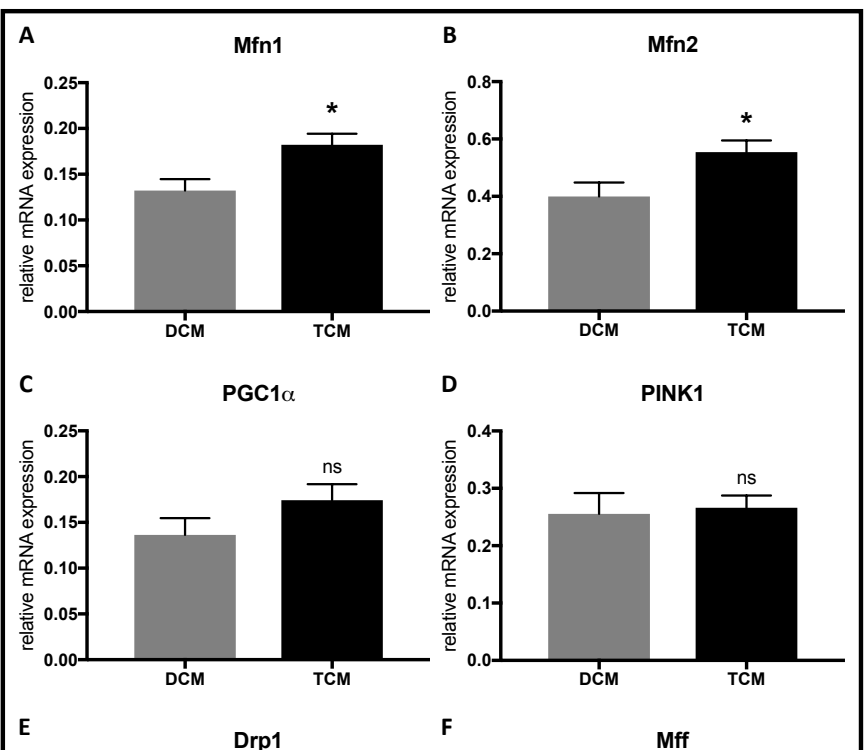

E
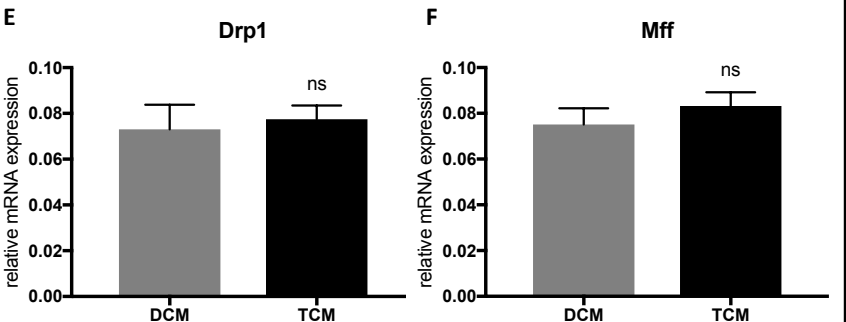

G
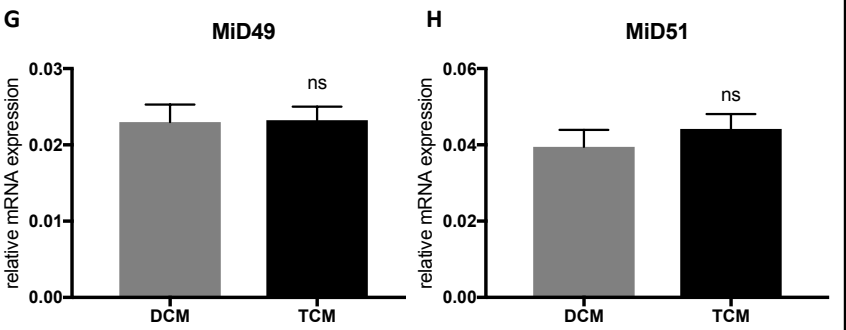

Fig. 1. Patients with tachycardiomyopathy show enhanced mRNA expression of mitochondrial fusion proteins. Relative mRNA expression levels in endomyocardial biopsies of patients with tachycardiomyopathy (TCM) and dilated cardiomyopathy (DCM) are shown, including mitochondrial fusion proteins Mfn1 (A), Mfn2 (B), and the upstream regulator PGC1 $\alpha$ (C), as well as PINK1 (D). Drp1 (E), MFF (F), MiD49 (G), and MiD51 (H) are involved in mitochondrial fission. * indicates $\mathrm{p}<0.05$, ns indicates $\mathrm{p}>0.05$. 1 and 2 have been found to be predominantly regulated at the transcription level [2].

Furthermore, intensive exercise training in mice has been associated with enhanced fusion and an increase of mitochondrial size, which is in line with the data we present [4]. Interestingly, mitochondria in DCM have been described to be significantly smaller than in control biopsies, advocating a pathophysiologically different mechanisms [5]. PGC1 $\alpha$, as a known upstream regulator, showed no significant regulation, which could indicate that other regulating factors or pathways could play a role in the increase of Mfn1/2 mRNA expression in TCM. 


\section{Cellular Physiology and Biochemistry}

Cell Physiol Biochem 2019;52:435-438

\begin{tabular}{l|l}
\hline DOI: 10.33594/000000031 & ( 2019 The Author(s). Published by
\end{tabular}

Heinzmann et al.: Mitochondrial Dynamics in TCM

Analyses of pivotal fission proteins Drp1, MFF, MiD49, and MiD51 did not reveal mRNA expression differences, suggesting that mRNA upregulation described above may be specific for TCM.

Elimination of mitochondria through mitophagy is dependent upon reduced degradation of PINK1 and Fis1. Neither PINK1 nor Fis1 expression was found to be regulated in TCM compared to DCM.

The presented results give first insight into underlying mechanisms of altered mitochondrial dynamics in patients with TCM, displaying the EMID-sign. Further analyses are needed to elucidate regulation via protein modification including phosphorylation, ubiquitination, and SUMOylation in these patients.

\section{Acknowledgements}

The study protocol has been approved by the committee on human research of the Eberhard-Karls University of Tübingen.

This study was funded by the DFG (German Research Foundation) Klinische Forschungsgruppe 274: Platelets - Molecular Mechanisms and Translational Implications and the Collaborative Research Center Transregio 240 (project number: 374031971).

\section{Disclosure Statement}

The authors declare no conflicts of interest.

\section{References}

1 Mueller KAL, Heinzmann D, Klingel K, Fallier-Becker P, Kandolf R, Kilias A, Walker-Allgaier B, Borst O, Kumbrink J, Kirchner T, Langer H, Geisler T, Schreieck J, Gramlich M, Gawaz M, Seizer P: Histopathological and Immunological Characteristics of Tachycardia-Induced Cardiomyopathy. J Am Coll Cardiol 2017;69:2160-2172.

2 Marin-Garcia J, Akhmedov AT: Mitochondrial dynamics and cell death in heart failure. Heart Fail Rev 2016;21:123-136.

3 Murphy E, Ardehali H, Balaban RS, DiLisa F, Dorn GW 2nd, Kitsis RN, Otsu K, Ping P, Rizzuto R, Sack MN, Wallace D, Youle RJ, American Heart Association Council on Basic Cardiovascular Sciences CoCC, Council on Functional G, Translational B: Mitochondrial Function, Biology, and Role in Disease: A Scientific Statement From the American Heart Association. Circ Res 2016;118:1960-1991.

4 Coleman R, Silbermann M, Gershon D, Reznick AZ: Giant mitochondria in the myocardium of aging and endurance-trained mice. Gerontology 1987;33:34-39.

5 Tashiro A, Masuda T, Segawa I: Morphometric comparison of mitochondria and myofibrils of cardiomyocytes between hypertrophic and dilated cardiomyopathies. Virchows Arch A Pathol Anat Histopathol 1990;416:473-478. 